

\title{
Modelling inspection and replacement quality for a protection system
}

\author{
Alexandre R. Alberti ${ }^{a}$; Cristiano A.V. Cavalcante ${ }^{i}$; Philip Scarf ${ }^{b}$; André L.O. Silva ${ }^{c}$ \\ ${ }^{a}$ Department of Production Engineering, Federal University of Pernambuco, Brazil. \\ ${ }^{b}$ Salford Business School, University of Salford, Manchester \\ ${ }^{c}$ COMPESA, Companhia Pernambucana de Saneamento, Brazil.
}

\begin{abstract}
An inspection and replacement policy for a protection system is described by a mathematical model that incorporates multiple aspects of maintenance quality. A three-state component failure model is assumed, with a defective state preceding failure. The quality of maintenance intervention is modelled by supposing that inspections may misclassify defects (false positives and false negatives) and further that an inspection may induce a defect. The quality of replacement is modelled by supposing that a component arises from a heterogeneous population, composed of weak and strong items and with the mixing parameter determining quality. Isolation valves used in water distribution systems motivate the model development, and a case study is considered in this context. We evaluate the impact of these aspects of the quality of maintenance upon cost and production losses. Defect induction is found to be a key determinant of the cost-optimal policy. The proposed model allows us to verify conditions that justify investment in higher quality maintenance, and thus to provide guidance for prioritization of this investment.
\end{abstract}

KEYWORDS: maintenance; preparedness system; water; distribution network.

\section{INTRODUCTION}

Protection systems, such as fire alarms, defibrillators, flood barriers and military defence systems, are typically inactive for much of their lives, and their activation is only required in specific situations such as emergencies (demand events). Consequently, such systems present hidden failures that are only detected in the case of an emergency, or through routine inspections. Moreover, an emergency event not contained by a failed protection system can have severe consequences, so concerns about the adequate maintenance of these systems are well justified. To ensure a high level of availability of these systems, they should be subject to regular inspection. Such concerns and the consequent inspection processes have been modelled (Vaurio, 1995, 1999; Jia and Christer, 2002; Cavalcante et al., 2011; Berrade et al., 2012, 2013, 2015).

In general, the maintenance requirements (Dwight et al., 2012) for a system are developed in three steps. First, the scope of the maintenance policy is established by consideration of the types of actions that are appropriate for the system. Secondly, the frequency at which such actions should be executed is determined. This can be accomplished through subjective evaluation, as with the reliability centered maintenance methodology (Selvik and Aven, 2011), or through the application of mathematical models (Scarf, 1997) that aim to optimize a suitable evaluation criterion (Aven and Jensen, 1999). Finally, the requirement for resources to carry out the maintenance is specified. What is often ignored in this process is the impact of the quality of the resources available upon the efficacy of maintenance. 
Some mathematical studies have sought to model particular aspects of maintenance quality. For example, Scarf et al. (2009) model the quality of component replacement by supposing that components are heterogeneous, that is, arising from a mixed population of strong items and weak items, the latter subject to premature failure. Cavalcante et al. (2011), Berrade et al. (2012, 2015), Scarf and Cavalcante (2012) and De Jonge et al. (2015) share this same notion. The source of heterogeneity may stem from variation in manufacturing or refurbishment of components, or from variation in the quality of the installation of components, or both. To model inspection quality, studies consider classification errors (false alarms and false negatives) (e.g. Okumura et al., 1996; Dagg and Newby, 1998; Berrade et al.; 2012, 2015; Cavalcante et al., 2017; Driessen et al., 2017). Other studies consider the possibility that inspections themselves induce faults or defects into the system (Scarf and Cavalcante; 2012; Flage, 2014).

The objectives of this study are to evaluate the impact of these different aspects of maintenance quality on the performance of a protection system, to determine the conditions that warrant investment in higher quality maintenance, and to inform decision-making about priorities for improvement. Such aspects would in reality include matters relating to specialized training and available technologies. To meet these objectives, we present a mathematical model that incorporates these three aspects of inspection and replacement quality (classification errors, defect induction, and component heterogeneity). Classification errors and defect induction relate to the quality of inspection. Component heterogeneity (variable quality of components) relates to quality of replacement which itself may be viewed as a kind of "defect induction" at replacement. We use the delay time concept (Christer, 1999) as a framework for our study and model a single-component protection system that is subject to periodic inspection and preventive replacement. Our approach is innovative because (i) we model these three aspects of maintenance quality simultaneously, and (ii) we motivate the study using a real case.

The case study itself considers the maintenance of isolation valves used in a water distribution system. We study: the sensitivity of the policy to parameters that characterize maintenance quality; the effect of ignorance of some aspect of maintenance quality; and the cost-benefit of higher quality maintenance in the long term. The model we develop has general applicability, although it is important to map the operational aspects of the system when applying the model to a real-life scenario. For example, maintenance of the protection system may imply downtime of the protected system (e.g. an emergency brake system) or may not (e.g. fire alarm).

This paper is structured as follows. Next we develop the model, its assumptions, and the optimization criterion. In section 3, we describe the case study and the contextual setting that motivates the model development, present our results and a discussion of those. Finally, we conclude with some remarks about the study.

\section{THE MAINTENANCE QUALITY MODEL}

We consider a protection system that is a one-component system (a component in a socket that together perform an operational function) (Ascher and Feingold, 1984). The system is subject to a three-state failure process: the defective state precedes the failed state. Inspection reveals the state of the system - this is the delay time model (Christer, 1999) — although the inspection signal is subject to error. When the system is defective, it is still able to perform its operational function. When a component is replaced, the system is renewed. Downtime is defined as the period when the protection system is in the failed state. 
While a two-state failure process is adequate to model the inspection of a protection system (e.g. Vaurio, 1999; Berrade et al., 2012; 2015), we consider a three-state model because it provides more information when detection of the defective state is possible and we wish to model classification errors related to this state. These errors are false positives (inspection indicates the system is defective when its true state is good) and false negatives (inspection indicates the system is good when its true state is defective). We assume that when the system is failed, inspection indicates the system is failed with probability 1 . Inspections may also induce a defect. This occurs when an inspection changes the state of the system from good to defective.

From new, $M$ inspections are scheduled with inspection interval $T$. When an inspection indicates a defect or failure, the component is replaced. Replacement is scheduled at the $M$-th inspection regardless of the state of the system (see Vaurio, 1999). The policy thus has two decision variables $(M$ and $T)$, and pure inspection $(M=\infty)$ and age-based replacement $(M=1)$ are special cases.

The model is established in a general context where there are demand events of different types (of different levels of severity in practice), and the consequent costs and production losses are different. Also, demands incur a cost and production loss even when the protection system is operational. However, when the protection system is failed on demand, additional costs (in the manner of penalty costs) (Christer and Scarf, 1994) and losses are incurred. Costs and losses are accumulated in two separate decision criteria.

\subsection{Notation}

\begin{tabular}{ll}
\hline$X$ & time to defect arrival - random variable \\
$H$ & delay-time, that is, the time or sojourn in the defective state - random variable \\
$\mathrm{Z}$ & State of the protection system, $\mathrm{Z} \in\{\mathrm{G}, \mathrm{D}, \mathrm{F}\}$, and the operational state is $\mathrm{O}=\{\mathrm{G}, \mathrm{D}\}$ \\
$f_{X}, F_{X} R_{X}$ & For $X$, the probability density, distribution and reliability functions \\
$f_{H}, F_{H} R_{H}$ & For $H$. the probability density, distribution and reliability functions \\
$M$ & scheduled number of inspections until replacement \\
$T$ & time interval between cessation of one inspection and commencement of the next \\
$E C$ & expected total cost of inspection and replacement in a renewal cycle \\
$E L$ & expected length of a renewal cycle (cycle length) \\
$E D$ & expected downtime in a renewal cycle \\
$E S$ & expected total duration of inspection and replacement in a renewal cycle \\
$E S h$ & expected shortage in a renewal cycle \\
$P$ & mixture parameter (proportion of weak items) \\
$W$ & probability of false positive at an inspection \\
$Q$ & probability of false negative at an inspection \\
$R$ & probability of defect induction at an inspection \\
$S_{\mathrm{I}}$ & time required to complete an inspection \\
$S_{\mathrm{Z}}$ & time required to replace a component when the system state is $\mathrm{Z}, \mathrm{Z} \in\{\mathrm{G}, \mathrm{D}, \mathrm{F}\}$ \\
$c_{\mathrm{I}}$ & cost of an inspection \\
$c_{\mathrm{Z}}$ & cost of a replacement when the system state is $\mathrm{Z}, \mathrm{Z} \in\{\mathrm{G}, \mathrm{D}, \mathrm{F}\}$ \\
$C_{\infty}$ & long-run cost per unit of time, or cost-rate \\
$S h_{\infty}$ & long-run production loss per unit of time, or loss-rate \\
$k$ & index specifying the type of demand \\
$\mu_{k}$ & rate of occurrence of demand of type $k$ \\
$C U D_{k}$ & extra costs incurred due to a type $k$ unmet demand
\end{tabular}


$D_{\mathrm{O}} \quad$ production loss-rate on demand when system is operational $(\mathrm{Z} \in\{\mathrm{G}, \mathrm{D}\}=\mathrm{O})$

$D_{\mathrm{F}} \quad$ production loss-rate on demand when system is $\mathrm{F}$

$D_{\mathrm{M}} \quad$ production loss-rate during maintenance

$T_{k, \mathrm{O}} \quad$ Downtime when type $k$ demand occurs and system is operational $(\mathrm{Z} \in \mathrm{O})$.

$T_{k, \mathrm{~F}} \quad$ Downtime when type $k$ demand occurs and system is $\mathrm{F}$

\subsection{List of model assumptions}

1. The system is renewed when the component is replaced.

2. The time to defect arrival $X$ has a mixture distribution $f_{X}(x)=p f_{1}(x)+(1-p) f_{2}(x)$.

3. The time to defect arrival $X$ and delay time $H$ are statistically independent.

4. A defect induced at an inspection can only be detected at a subsequent inspection.

5. Defect induction does not occur when the protection system is operated on demand.

6. There are no classification errors regarding identification of a failed state, that is $\operatorname{Pr}(\mathrm{F}, \mathrm{G})=\operatorname{Pr}(\mathrm{G}, \mathrm{F})=0$, where $\operatorname{Pr}\left(\mathrm{Z}_{1}, \mathrm{Z}_{2}\right)$ is the probability that an inspection reports the system state as $Z_{1}$ when the true system state is $Z_{2}$.

7. The protection system does not age during inspection.

8. At the scheduled replacement at age $M T$ the protection system is inspected. We justify this on the basis that the system operator would wish to know the system state at the point of replacement.

9. Demand events (external to the protection system itself) of type $k$ arise according to a Poisson process with rate $\mu_{k}$, (independent), and an unmet demand of type $k$ (protection system failed) incurs a cost $C U D_{k} \quad k=1, \ldots, m$.

10. Operation of the protection system on demand takes non-negligible time $T_{k, \mathrm{Z}}$ that depends on the demand type and the state of the protection system. During this time downtime accrues. Further, production is lost at rate $D_{k, Z}$ during this downtime. Also, we assume that the downtime when the system is good or defective are the same, so that $T_{k, \mathrm{O}}=T_{k, \mathrm{G}}=T_{k, \mathrm{D}}<T_{k, \mathrm{~F}}$, and the losses do not depend on the demand type or whether the system is good or defective, so that $D_{k_{1}, \mathrm{Z}}=D_{k_{2}, \mathrm{Z}}=D_{\mathrm{Z}}$ for all $k_{1}$ and $k_{2}$ and $\mathrm{Z}$, and $D_{\mathrm{O}}=D_{\mathrm{G}}=D_{\mathrm{D}}<D_{\mathrm{F}}$.

11. The durations of an inspection and a repair are known constants

12. The protection system is available during inspection and replacement, so that downtime is not accruing. Thus we suppose that maintenance execution times are non-negligible but that in the event of a demand for the system during maintenance the operational function of the protection system is available, via some alternate provision. However, production is lost at rate $D_{\mathrm{M}}$ during maintenance.

\subsection{Model development}

Calculations proceed by determining the probabilities of all possible disjoint replacement (renewal) events, which we call scenarios (Figure 1), and their respective costs and durations. To verify that these are disjoint and exhaustive (so that these events partition the sample space), we check that their probabilities sum to 1 . Note that some scenarios arise only when $M>1$.

- Scenario 1 - replacement at a true positive inspection $(M>1)$

A defect arises naturally in the $j$-th interval between inspections or is induced at the $j$-th inspection, and the $i$-th inspection $(j \leq i<M)$ is positive (the inspection signal is $\mathrm{D}$ ), and the component is replaced. This occurs with probability 


$$
\begin{aligned}
& P_{1}(T, i)=\sum_{j=1}^{i}(1-r)^{j-1}(1-w)^{j-1} q^{i-j}(1-q) \int_{(j-1) \cdot T}^{j . T} f_{X}(x) R_{H}(i T-x) d x \\
& +\sum_{j=1}^{i-1} R_{X}(j T)(1-r)^{j-1} r(1-w)^{j} q^{i-j-1}(1-q) R_{H}\{(i-j) T\} \\
& P_{1}(T, 1)=(1-q) \int_{0}^{T} f_{X}(x) R_{H}(T-x) d x
\end{aligned}
$$

The maintenance cost is:

$$
E C_{1}(T, M)=\sum_{i=1}^{M-1}\left(i c_{\mathrm{I}}+c_{\mathrm{D}}\right) P_{1}(T, i),
$$

the cycle length is

$$
E L_{1}(T, M)=\sum_{i=1}^{M-1}\left\{S_{\mathrm{D}}+i\left(T+S_{\mathrm{I}}\right)\right\} P_{1}(T, i),
$$

the downtime is $E D_{1}(T, M)=0$, and the time duration of maintenance actions (inspection and replacement) is

$$
E S_{1}(T, M)=\sum_{i=1}^{M-1}\left(i S_{\mathrm{I}}+S_{\mathrm{D}}\right) P_{1}(T, i) .
$$

\section{Scenario}

$\begin{aligned} & \text { Replacement at a true } \\ & \text { positive inspection }\end{aligned}$
$\begin{aligned} & \text { Replacement on failure } \\ & \text { following a sequence of } \\ & \text { false negatives }\end{aligned}$
$\begin{aligned} & \text { Replacement on failure } \\ & \text { following no false } \\ & \text { negatives }\end{aligned}$

Failure before the first

4 inspection

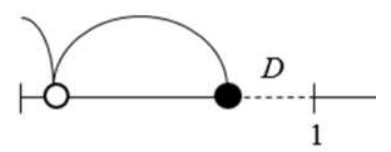

Replacement at a false positive inspection
5

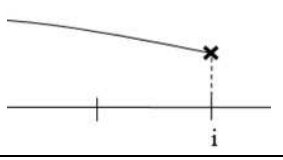

Replacement of a defective system at MT
$6 a$

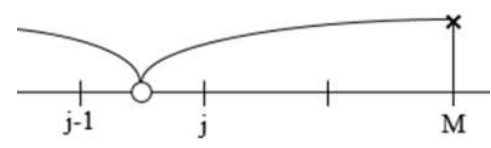

$6 b$

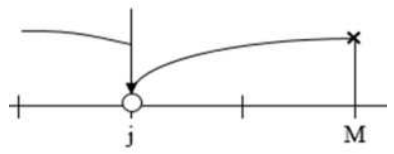


Replacement of a good system at $M T$
7

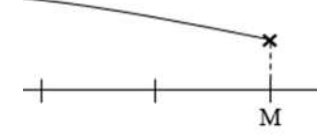

Figure 1. Schematics for each of the renewal scenarios; o defect arrival,

failure.

- $\quad$ Scenario 2 - replacement on failure following a sequence of false negatives $(M>1)$

A defect arises in the $j$-th interval between inspections or is induced at the $j$-th inspection, is not detected at subsequent inspections, and fails, and the component is replaced (renewal) at the $(i+1)$-th inspection $(i<M)$. This occurs with probability

$$
\begin{array}{ll}
P_{2}(T, i)=\sum_{j=1}^{i}(1-r)^{j-1}(1-w)^{j-1} q^{i-j+1} \int_{(j-1) . T}^{j . T} \int_{(i . T-x)}^{((i+1) \cdot T-x)} f_{H}(h) f_{X}(x) d h d x & \\
+\sum_{j=1}^{i-1} R_{X}(j T)(1-r)^{j-1} r(1-w)^{j} q^{i-j} \int_{(i-j) T}^{(i+1-j) T} f_{h}(h) d h, & \text { (for } i>1), \\
P_{2}(T, i)=q \int_{0}^{T} \int_{(T-x)}^{(2 . T-x)} f_{H}(h) f_{X}(x) d h d x, & \text { (for } i=1) .
\end{array}
$$

The maintenance cost is

$$
E C_{2}(T, M)=\sum_{i=1}^{M-1}\left\{(i+1) c_{\mathrm{I}}+c_{\mathrm{F}}\right\} P_{2}(T, i),
$$

and cycle length is

$$
E L_{2}(T, M)=\sum_{i=1}^{M-1}\left\{(i+1)\left(T+S_{\mathrm{I}}\right)+S_{\mathrm{F}}\right\} P_{2}(T, i) .
$$

The downtime for each $i$ is

$$
\begin{array}{ll}
E D_{2, i}(T, i)= & \\
\sum_{j=1}^{i}(1-r)^{j-1}(1-w)^{j-1} q^{i-j+1} \int_{(j-1) T}^{j . T} \int_{(i T-x)}^{((i+1) T-x)}\{T(i+1)-(x+h)\} f_{H}(h) f_{X}(x) d h d x & \text { (for } i>1) \\
+\sum_{j=1}^{i-1} R_{X}(j T)(1-r)^{j-1} r(1-w)^{j} q^{i-j} \int_{(i-j) T}^{(i+1-j) T}\{T(i+1)-(j T+h)\} f_{H}(h) d h, & \\
E D_{2,1}(T, i)=q \int_{0}^{T} \int_{(T-x)}^{(2 T-x)}\{2 T-(x+h)\} f_{H}(h) f_{X}(x) d h d x, & \text { (for } i=1),
\end{array}
$$

so that the downtime is

$$
E D_{2}(T, M)=\sum_{i=1}^{M-1} E D_{2, i}(T, i) .
$$

The time duration of maintenance actions is

$$
E S_{2}(T, M)=\sum_{i=1}^{M-1}\left\{(i+1) S_{\mathrm{I}}+S_{\mathrm{F}}\right\} P_{2}(T, i) .
$$


- $\quad$ Scenario 3 - replacement on to failure following no false negatives $(M>1)$

A defect arises naturally after the $i$-th inspection or is induced at the $i$-th inspection and failure occurs before the $(i+1)$-th inspection $(i<M)$. This occurs with probability

$$
P_{3}(T, i)=(1-r)^{i}(1-w)^{i} \int_{i . T}^{(i+1) \cdot T} F_{H}\{(i+1) T-x\} f_{X}(x) d x+R_{X}(i T)(1-r)^{i-1} r(1-w)^{i} F_{H}(T)
$$

for all $i$, and the maintenance cost is

$$
E C_{3}(T, M)=\sum_{i=1}^{M-1}\left\{(i+1) c_{\mathrm{I}}+c_{\mathrm{F}}\right\} P_{3}(T, i),
$$

and the cycle length is

$$
E L_{\mathcal{3}}(T, M)=\sum_{i=1}^{M-1}\left\{(i+1)\left(T+S_{\mathrm{I}}\right)+S_{\mathrm{F}}\right\} P_{3}(T, i) .
$$

The downtime for each $i$ is

$$
\begin{aligned}
& E D_{3, i}(T, i)=(1-r)^{i}(1-w)^{i} \int_{i T}^{(i+1) T} \int_{0}^{((i+1) T-x)}\{(i+1) T-(x+h)\} f_{H}(h) f_{X}(x) d h d x \\
& +R_{X}(i T)(1-r)^{i-1} r(1-w)^{i} \int_{0}^{T}(T-h) f_{H}(h) d h
\end{aligned}
$$

for all $i$, and so the downtime is

$$
E D_{3}(T, M)=\sum_{i=1}^{M-1} E D_{3, i}(T, i)
$$

The time duration of maintenance actions is

$$
E S_{3}(T, M)=\sum_{i=1}^{M-1}\left\{(i+1) S_{\mathrm{I}}+S_{\mathrm{F}}\right\} P_{3}(T, i) .
$$

- Scenario 4 -failure before the first inspection (any $M$ )

Failure occurs before the first inspection due to a defect that arises naturally. This occurs with probability

$$
P_{4}(T)=\int_{0}^{T} f_{x}(x) F_{H}(T-x) d x .
$$

The maintenance cost is $E C_{4}(T)=\left(c_{\mathrm{I}}+c_{\mathrm{F}}\right) P_{4}(T)$, the cycle length is $E L_{4}(T)=\left(T+S_{\mathrm{I}}+S_{\mathrm{F}}\right) P_{4}(T)$, the downtime is

$$
E D_{4}(T)=\int_{0}^{T} \int_{0}^{(T-x)}\{T-(x+h)\} f_{H}(h) f_{X}(x) d h d x,
$$

and the time duration of maintenance actions is $E S_{4}(T)=\left(S_{\mathrm{I}}+S_{\mathrm{F}}\right) P_{4}(T)$.

- $\quad$ Scenario 5 - replacement at a false positive $(M>1)$

At the $i$-th inspection $(i<M)$ a false positive occurs and the system is renewed with probability

$$
P_{5}(T, i)=(1-r)^{i-1}(1-w)^{i-1} w R_{X}(i T)
$$

for all $i<M$. The maintenance cost is 


$$
E C_{5}(T, M)=\sum_{i=1}^{M-1}\left(i c_{\mathrm{I}}+c_{\mathrm{G}}\right) P_{5}(T, i)
$$

the cycle length is

$$
E L_{5}(T, M)=\sum_{i=1}^{M-1}\left\{i\left(T+S_{\mathrm{I}}\right)+S_{\mathrm{G}}\right\} P_{5}(T, i),
$$

the downtime is $E D_{5}(T, M)=0$, and time duration of maintenance actions is

$$
E S_{5}(T, M)=\sum_{i=1}^{M-1}\left(i S_{\mathrm{I}}+S_{\mathrm{G}}\right) P_{5}(T, i) .
$$

- $\quad$ Scenario 6 - Replacement of a defective system at MT (any M)

A defect arises naturally in the $j$-th interval between inspections or is induced at the $j$-th inspection and survives until the $M$-th inspection. This occurs with probability

$$
\begin{aligned}
& P_{6}(T, M)=\sum_{j=1}^{M}(1-r)^{j-1}(1-w)^{j-1} q^{M-j} \int_{(j-1) T}^{j T} f_{X}(x) R_{H}(M T-x) d x \\
& +\sum_{j=1}^{M-1} R_{X}(j T)(1-r)^{j-1} r(1-w)^{j} q^{M-j-1} R_{H}\{(M-j) T\}, \\
& P_{6}(T, M)=\int_{0}^{T} f_{X}(x) R_{H}(M T-x) d x,
\end{aligned}
$$

$($ for $M>1)$,

(for $M=1)$,

noting that if $M=1$ no defect can be induced. The maintenance cost is $E C_{6}(T, M)=\left(c_{d}+M c_{\mathrm{I}}\right) P_{6}(T, M)$, the cycle length is $E L_{6}(T, M)=\left\{M\left(T+S_{\mathrm{I}}\right)+S_{\mathrm{D}}\right\} P_{6}(T, M)$, the downtime is $E D_{6}(T, M)=0$, and the duration of maintenance actions is $E S_{6}(T, M)=\left(M S_{\mathrm{I}}+S_{\mathrm{D}}\right) P_{6}(T, M)$.

- $\quad$ Scenario 7 - replacement of a good system at MT (any $M$ )

In this scenario, a defect does not arise (naturally or induced) before $M T$ and the system is replaced, with probability $P_{7}(T, M)=(1-r)^{M-1}(1-w)^{M-1} R_{X}(M T)$. The maintenance cost is $E C_{7}(T, M)=\left(c_{\mathrm{G}}+M c_{\mathrm{I}}\right) P_{7}(T, M)$, the cycle length is $E L_{7}(T, M)=\left\{M\left(T+S_{\mathrm{I}}\right)+S_{\mathrm{G}}\right\} P_{7}(T, M)$, the downtime is $E D_{7}(T, M)=0$, and the time duration of maintenance actions is $E S_{7}(T, M)=\left(M S_{\mathrm{I}}+S_{\mathrm{G}}\right) P_{7}(T, M)$.

\subsection{Determining the cost-rate}

The expectation of a quantity of interest $E Q(T, M)$ is $\sum_{s=1}^{7} E Q_{s}(T, M)$, the sum of the contributions to the expectation over each of the scenarios, where $E Q$ corresponds in turn to: the expected maintenance cost in a renewal cycle, $E C$; the cycle length, $E L$; the expected downtime in a renewal cycle, $E D$; and the expected duration of maintenance actions in a renewal cycle, ES. Then, considering additionally the expected rate of accumulation of cost of unmet demand when the system is down, $\sum_{k=1}^{m} \mu_{k} C U D_{k}$, the cost-rate (our optimization criterion) is

$$
C_{\infty}(T, M)=\frac{E C(T, M)+E D(T, M) \sum_{k=1}^{m} \mu_{k} C U D_{k}}{E L(T, M)} .
$$




\subsection{Auxiliary criterion}

Additionally we consider the loss-rate (the long-run production loss per unit time). This is expressed generally, although in the context of the case study that follows we regard these "production losses" as the loss of water supply to customers of the distribution network. In general the total expected loss in a renewal cycle is

$$
\begin{aligned}
& E S h(T, M)=\{E L(T, M)-E D(T, M)-E S(T, M)\} \sum_{k=1}^{m} \mu_{k} T_{k, \mathrm{O}} D_{\mathrm{O}} \\
& \quad+E D(T, M) \sum_{k=1}^{m} \mu_{k} T_{k, \mathrm{~F}} D_{\mathrm{F}}+E S(T, M) D_{\mathrm{M}} .
\end{aligned}
$$

Note that the three terms in this expression relate to losses due to demands that occur when the system is operational, losses due to demands that occur when the system is failed, and losses due to maintenance of the system, respectively. The loss rate is then

$$
S h_{\infty}(T, M)=\frac{E S h(T, M)}{E L(T, M)} .
$$

\subsection{Adapting the model to other contexts}

Depending on the context of application, assumption 12 deserves special attention. In Berrade et al. (2012), this assumption takes the opposite form (during replacement the protection system is down), but to adapt our model to this opposite assumption, it suffices to calculate the expected downtime as $(E D+E S)$. Assumption 7 can relaxed by considering $T$ as including $S_{\mathrm{I}}\left(T>S_{\mathrm{I}}\right)$ and removing $S_{\mathrm{I}}$ from the expressions for $E L$. The model presented can also be modified for protection systems in simpler contexts: components arising from homogeneous population, $p=0$; no classification errors, $w=q=$ 0 ; no defect induction, $r=0$; perfect inspection, $w=q=r=0$. Further, by defining $w=0$ and $q=1$ we model renewal only on failure, assuming no error in identifying the failed state. Finally, when the system has only two possible states (operational and failed) and is subject to perfect inspection, it suffices to define $w=q=r=0$ in the scenarios 3,4 and 7 and setting $F_{H}(h)=1$ for all $h$.

\section{THE CASE OF ISOLATION VALVES}

\subsection{Context}

The impact of isolation valves upon the reliability of water distribution systems (Kim and Mays, 1994) depends on the design of the isolation system and the reliability of the valves themselves. Many studies have considered the former (e.g., Walski, 1993; Jun et al., 2007; Giustolisi and Savic, 2010) but few consider the latter (Liu et al., 2017). Isolation valves are protection systems. Their operation is required to isolate part of the network (segment) only in the event of, for example, leakage, repair, or contamination. When a demand event occurs (on demand) and the required valves are fully functional, a segment is isolated with little delay, leaving only a limited number of clients without service and avoiding greater damage to the network. On the other hand, if a valve is failed (not functional) on demand, isolation is delayed causing greater loss of supply and, depending on the operational pressure in the segment, flooding and erosion (Figure 2), and adjacent segments must be isolated, leaving a greater number of customers without service.

The function of isolation valves is even more critical for controlling contamination events, which have a greater impact on service disruption, environmental damage, and cost. A complicating aspect is that isolation system design (the location of isolation valves in the network) renders some values more critical than others, and so the maintenance requirements may vary across identical valves. 
An isolation valve is regarded as failed when it cannot be closed. In common with mechanical equipment, the physical condition or "state" of a valve declines over time due to factors such as corrosion, fatigue, wear and accumulation of debris, the deterioration rate depending on the specific operational conditions of the valve (Marlow et al., 2012). In our study for a Brazilian sanitation company, the most failure-prone component is the reduction mechanism of the valve activation system. This wears relatively quickly and it is more sensitive to factors related to maintenance quality. Such components are used in valves of large nominal diameter and high operating pressure. Furthermore, in practice, prior to failure, the valve presents as defective, that is, even though it can fulfill its operational function, it shows warning signs of imminent failure such as resistance to closure or small passage of water on closure. Information about the state of the valve can be obtained at inspection.

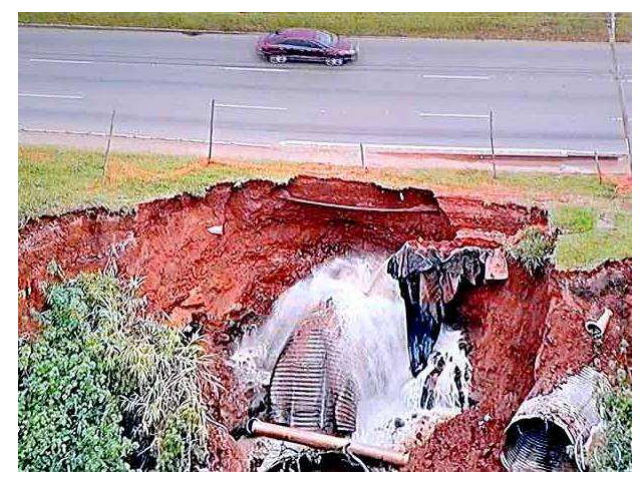

Figure 2. Major erosion resulting from a broken water pipeline in Brazil.

At an inspection, closure of the valve is attempted by an inspector and downstream water-flow is measured. The impact of inspection upon the operation of the network is negligible. Repair of a valve on the other hand requires isolation of the valve from the network, causing the network (the protected system) to be partially unavailable. This partial unavailability is somewhat different to the notion of risk of a demand for the protection system not being met when it is subject to maintenance. Discussion with network engineers revealed some important aspects of inspection:

(1) On closure, low flow rate through the valve is difficult to detect. The pressure gauges show pressure differences when water flow is high enough to fill the pipe's transverse section, which happens when the valve is failed (classification error).

(2) The perception of the resistance when closing a valve (a characteristic of defective valves) varies among inspectors (classification error). Nonetheless, it is possible to determine when a valve cannot be fully closed (failed valve).

(3) The use of tools such as levers to close the valves more easily is common practice. This practice can damage components of the activation mechanism of the valve (Figure 3) (defect induction).

Points (1) and (2) suggest that classification errors occur when a valve is defective, but not when it is failed. Further, point (3) suggests that inspection can induce the defect state.

On operation of the valves on demand, the probability of an induced defect is practically zero, since a larger and better prepared crew is mobilized for such operations. In this way, better quality maintenance is more costly (e.g. Zyl, 2014). In the event of a demand or maintenance (here represented specially by the replacement of the gear reducer), a volume of water is not delivered to a group of customers (a shortage or loss), and the severity of this loss depends on when the event 
occurs, the values operated, and their operational states. Losses are calculated for a typical valve. The loss criterion, $S h_{\infty}$, is volume of water not supplied per unit of time.
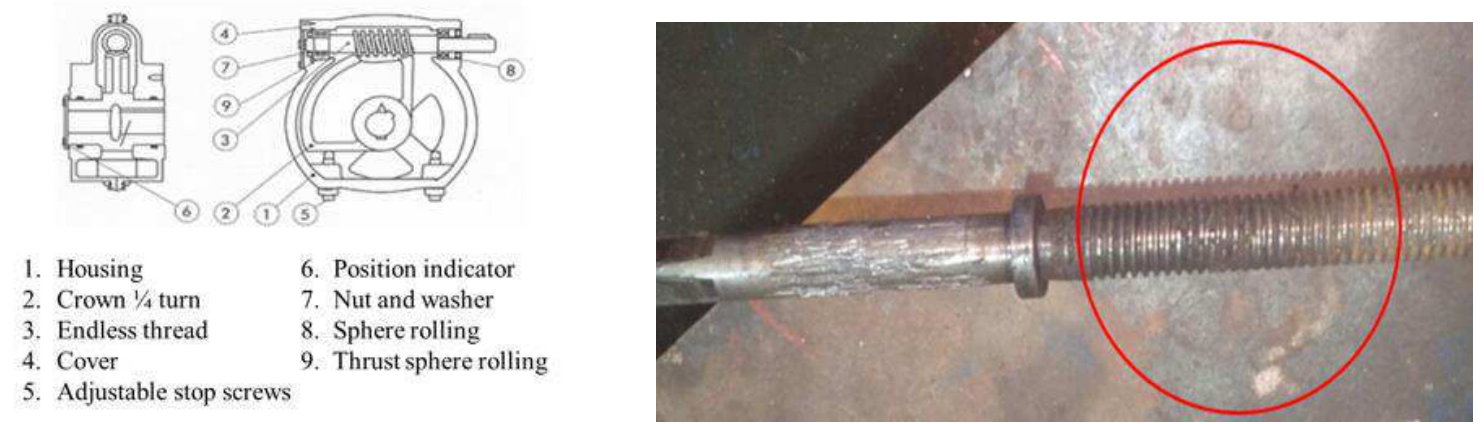

Figure 3. Left, schematic of a gear reducer in a butterfly valve, and right, endless thread damage due to use of excessive force in closure.

\subsection{Specification of the parameters}

We study an isolation valve in a water distribution network that is installed in a high-pressure pipeline, located in an easily accessible site. The segments adjacent to that valve have a high risk for contamination. The parameter estimates we use are based on expert knowledge obtained from the engineers of the network operator and maintainer. Approximately $10 \%$ of the valve components are either poorly installed or returned to inventory with a fault at installation $(p=0.1)$. Inspection parameters were set at $w=0.05$ (false positive), $q=0.3$ (false negative) and $r=0.05$ (defect induction). The unit of time is set to 1 year. Weibull distributions are used for $X$ (weak $\eta_{1}=1, \beta_{1}=2$; strong $\eta_{2}=4, \beta_{2}=3$ ) and $H$ is exponential with mean $\eta_{3}=0.5$.

The time durations of replacements are not state dependent and $S_{\mathrm{G}}=S_{\mathrm{D}}=S_{\mathrm{F}}=1$ day. The time duration of inspection is assumed to be zero $\left(S_{\mathrm{I}}=0\right)$.

We simplify demand and network segmentation into two kinds of demand (leaks and contamination) and known consequences, depending of the operational state, representing an average valve among those observed. Demands are Poisson processes (Goulter and Coals, 1986). We set the type 1 demand (leaks) rate to $\mu_{1}=3.042$ (one demand every 120 days), and the type 2 demand (contamination) rate to $\mu_{2}=0.5$ (one every 2 years). On demand, the loss-rate is $25.000 \mathrm{~m}^{3} /$ day for an operational valve $\left(D_{\mathrm{O}}\right)$ and $50.000 \mathrm{~m}^{3} /$ day for a failed valve $\left(D_{\mathrm{F}}\right)$. The downtimes for operational valves are 1 day for a type 1 demand $\left(T_{1, \mathrm{O}}\right)$ and 2 days for type 2 demand $\left(T_{2, \mathrm{O}}\right)$, and for failed valves they are 2 days $\left(T_{1, \mathrm{~F}}\right)$ and 4 days $\left(T_{2, \mathrm{~F}}\right)$ respectively. For replacement, the loss-rate is $50.000 \mathrm{~m}^{3} /$ day ( $D_{\mathrm{M}}$ ) regardless of the state of the system at replacement.

Costs are relative to the cost of preventive replacement of a good valve, so that $c_{\mathrm{G}}=1$ unit. The other costs were: $c_{\mathrm{I}}=0.04$ (inspection), and $c_{\mathrm{D}}=1.4$ and $c_{\mathrm{F}}=2$ for replacement of defective and failed valves respectively. The extra costs resulting from an unmet demand are $C U D_{1}=20$ (leaks) and $C U D_{2}=50$ (contamination). These are penalty costs in the sense of Christer and Scarf (1994) relating to additional loss of supply, cost of containment, and other impacts (e.g. extensive damage to the network and the environment).

\subsection{Results and discussion}

The results are discussed in three parts. Firstly, we evaluate the effects of different aspects of maintenance quality upon optimal policy. Secondly, we evaluate the effects of ignoring these aspects 
of quality in turn, and thus demonstrate the utility of our general model. Finally, we investigate circumstances that would justify investing in higher quality maintenance.

\subsubsection{Evaluation of the effects of different aspects of maintenance quality}

The base case is highlighted on line 1 of Table 1 , and quality parameters are varied thereafter. In each case, we present the cost-optimal $(M, T)$ policy, the cost-optimal $(M=1, T)$ policy (age based replacement, $\mathrm{ABR}$ ) and the cost-optimal $(M=\infty, T)$ policy (pure inspection, PI). The secondary criterion, the loss or shortage is also presented.

In the base case, the ABR policy is globally optimal as the quality of inspection is such that inspection is detrimental. When we vary the maintenance quality parameters, the optimal policy appears to oscillate between ABR and PI or a policy near PI (MT policy for large $M$ ). We can see this effect in Figure 4, noting there that when $M=1$, the cost-rate does not depend on the value of the inspection parameters $(r, w$, and $q$ ) because there is no inspection in this case. The cost-optimality of PI would be doubtful however when there exist classification errors regarding failure, since downtime would not be limited by the length of the inspection interval. Detailed analysis of this point would require a different study. Further, looking at the optimal ABR policy, this is strongly influenced by the mixture parameter $p$ but cannot be influenced by the inspection quality parameters. This demonstrates that the $M T$ policy is interesting to study because it offers insights to aspects of quality that the simpler policies do not. Nonetheless, a policy that is simpler to implement (ABR)) might be used by the water distribution company. Furthermore, a block replacement policy (even simpler to implement) may be near cost-optimal, and so might be implemented.

Table 1. Comparison of optimal policies for various values of the quality parameters. $\mathrm{Sh}_{\infty}$ in $10^{5} \mathrm{~m}^{3}$.

\begin{tabular}{|c|c|c|c|c|c|c|c|c|c|c|c|c|c|c|c|c|}
\hline \multirow{2}{*}{\multicolumn{2}{|c|}{ Case }} & \multicolumn{4}{|c|}{ Quality parameters } & \multicolumn{5}{|c|}{ Optimal MT policy } & \multicolumn{3}{|c|}{$\begin{array}{l}\text { Optimal ABR } \\
\text { policy }(M=1)\end{array}$} & \multicolumn{3}{|c|}{$\begin{array}{l}\text { Optimal PI policy } \\
\qquad(M=\infty)\end{array}$} \\
\hline & & $p$ & $w$ & $q$ & $r$ & $M$ & $T$ & $M T$ & $C_{\infty} *$ & $S h_{\infty}$ & $T$ & $C_{\infty} *$ & $S h_{\infty}$ & $T$ & $C_{\infty} *$ & $S h_{\infty}$ \\
\hline 1 & Base case & 0.10 & 0.05 & 0.30 & 0.05 & 1 & 0.785 & 0.785 & 1.856 & 1.659 & 0.785 & 1.856 & 1.659 & 0.151 & 2.036 & 1.399 \\
\hline 2 & Worse $p$ & 0.15 & 0.05 & 0.30 & 0.05 & 1 & 0.703 & 0.703 & 2.070 & 1.734 & 0.703 & 2.070 & 1.734 & 0.149 & 2.086 & 1.412 \\
\hline 3 & Worse $p$ & 0.20 & 0.05 & 0.30 & 0.05 & 24 & 0.151 & 3.624 & 2.134 & 1.425 & 0.649 & 2.240 & 1.794 & 0.147 & 2.138 & 1.426 \\
\hline 4 & Better $p$ & 0.05 & 0.05 & 0.30 & 0.05 & 1 & 0.929 & 0.929 & 1.561 & 1.559 & 0.929 & 1.561 & 1.559 & 0.152 & 1.988 & 1.388 \\
\hline 5 & Best $p$ & 0 & 0.05 & 0.30 & 0.05 & 1 & 1.239 & 1.239 & 1.072 & 1.419 & 1.239 & 1.072 & 1.419 & 0.154 & 1.942 & 1.376 \\
\hline 6 & Worse $r$ & 0.10 & 0.05 & 0.30 & 0.10 & 1 & 0.785 & 0.785 & 1.856 & 1.659 & 0.785 & 1.856 & 1.659 & 0.150 & 2.936 & 1.525 \\
\hline 7 & Better $r$ & 0.10 & 0.05 & 0.30 & 0.03 & 20 & 0.162 & 3.240 & 1.644 & 1.353 & & 1.856 & 1.659 & 0.150 & 1.661 & 1.351 \\
\hline 8 & Better $r$ & 0.10 & 0.05 & 0.30 & 0.01 & 15 & 0.184 & 2.760 & 1.253 & 1.314 & 0.785 & 1.856 & 1.659 & 0.148 & 1.282 & 1.303 \\
\hline 9 & Best $r$ & 0.10 & 0.05 & 0.30 & 0 & 17 & 0.178 & 3.026 & 1.051 & 1.288 & 0.785 & 1.856 & 1.659 & 0.147 & 1.090 & 1.280 \\
\hline 10 & Worse $w$ & 0.10 & 0.10 & 0.30 & 0.05 & 1 & 0.785 & 0.785 & 1.856 & 1.659 & 0.785 & 1.856 & 1.659 & 0.180 & 2.180 & 1.466 \\
\hline 11 & Best $w$ & 0.10 & 0 & 0.30 & 0.05 & 1 & 0.785 & 0.785 & 1.856 & 1.659 & 0.785 & 1.856 & 1.659 & 0.124 & 1.861 & 1.312 \\
\hline 12 & Worse $q$ & 0.10 & 0.05 & 0.50 & 0.05 & 1 & & 0.785 & 1.856 & 1.659 & 0.785 & 1.856 & 1.659 & 0.138 & 2.239 & 1.422 \\
\hline 13 & Better $q$ & 0.10 & 0.05 & 0.10 & 0.05 & 1 & 0.785 & 0.785 & 1.856 & 1.659 & 0.785 & 1.856 & 1.659 & 0.164 & 1.878 & 1.379 \\
\hline 14 & Best $q$ & 0.10 & 0.05 & 0 & 0.05 & 20 & 0.178 & 3.560 & 1.805 & 1.371 & 0.785 & 1.856 & 1.659 & 0.172 & 1.810 & 1.369 \\
\hline 15 & Better $w$ and $q$ & 0.10 & 0.01 & 0.10 & 0.05 & 25 & 0.145 & 3.625 & 1.749 & 1.319 & 0.785 & 1.856 & 1.659 & 0.140 & 1.753 & 1.316 \\
\hline 16 & Best $w$ and $q$ & 0.10 & 0 & 0 & 0.05 & 25 & 0.146 & 3.650 & 1.656 & 1.295 & 0.785 & 1.856 & 1.659 & 0.140 & 1.659 & 1.291 \\
\hline 17 & Ignoring defects & 0.10 & 0 & 1 & 0.05 & 1 & 0.785 & 0.785 & 1.856 & 1.659 & 0.785 & 1.856 & 1.659 & 0.092 & 3.276 & 1.358 \\
\hline 18 & Ignoring defects, worse $r$ & 0.10 & 0 & 1 & 0.10 & 1 & 0.785 & 0.785 & 1.856 & 1.659 & 0.785 & 1.856 & 1.659 & 0.083 & 4.688 & 1.500 \\
\hline 19 & Ignoring defects, better $r$ & 0.10 & 0 & 1 & 0.01 & 14 & 0.133 & 1.862 & 1.687 & 1.321 & 0.785 & 1.856 & 1.659 & 0.086 & 1.775 & 1.255 \\
\hline 20 & Ignoring defects, best $r$ & 0.10 & 0 & 1 & 0 & 20 & 0.109 & 2.180 & 1.309 & 1.264 & 0.785 & 1.856 & 1.659 & 0.084 & 1.341 & 1.229 \\
\hline 21 & Perfect inspection & 0.10 & 0 & 0 & 0 & 20 & 0.164 & 3.280 & 0.818 & 1.197 & 0.785 & 1.856 & 1.659 & 0.134 & 0.838 & 1.175 \\
\hline 22 & Perfect maintenance & 0 & 0 & 0 & 0 & 15 & 0.192 & 2.880 & 0.749 & 1.201 & 1.239 & 1.072 & 1.419 & 0.130 & 0.800 & 1.165 \\
\hline
\end{tabular}


Focusing now on detail, we see (lines 1 to 5) that the smaller is $p$ the more competitive is ABR compared to PI. This is as expected since inspection is useful if early failures are likely and only if inspections themselves are effective (small classification errors) and efficient (no defect induction). Indeed for $r \geq 0.05$ the ABR policy is the recommended. This becomes even more evident when there is a combined effect of defect induction and false negatives (line 12): with no false negatives $(q=0)$, PI is preferred (line 14). The difference is small nonetheless, evidencing the greater impact of defect induction (lines 1 and 6-9, 0.01 change in $r$ has a 9\% effect on the cost-rate).

Variation in $w$ (false positives) (lines 1 and 10,11) has little effect on the MT policy and influences $T$ in PI (larger $T$ for larger $w$ ). Variation in $q$ (false negatives) (lines 1 and 12-14) we see the opposite (smaller $T$ for larger $q$ ). These observations concur with those in Berrade et al. (2012). These opposing effects appear to cancel one another (cases 15, 16). So defect induction is a key influencer of the cost-optimal policy. Regarding shortages (reliability criterion), more inspection leads to less loss of supply, and shortages are least when defect induction is least.

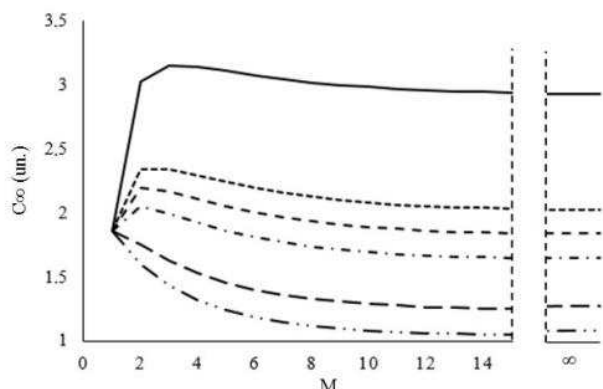

(a)

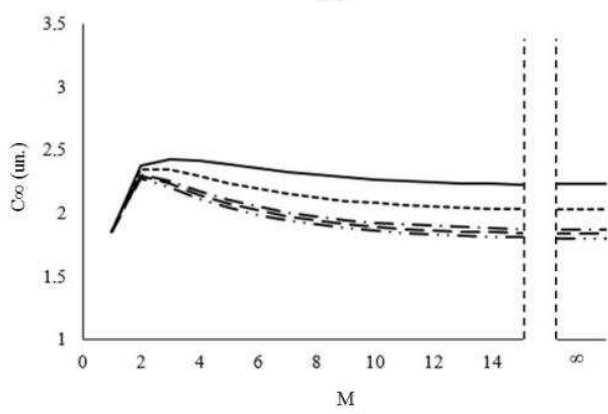

(c)

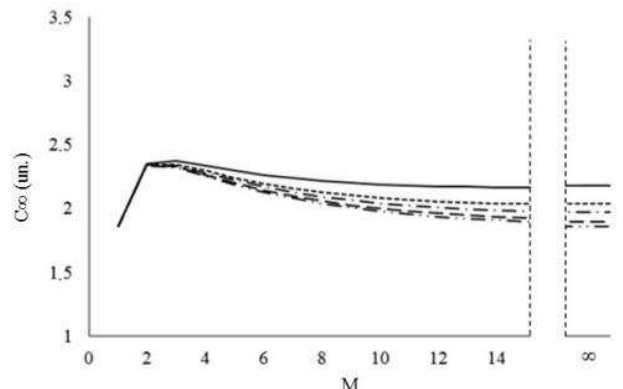

(b)

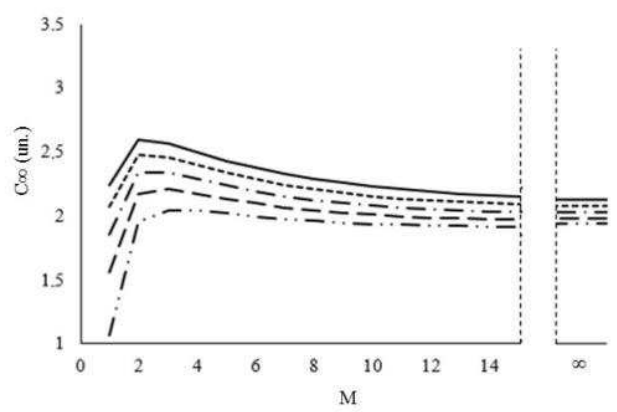

(d)

Figure 4. Cost-rate as a function of $M$. (a) $r$ varying, $r=0.1(-), r=0.05(--), r=0.04(---), r=0.03$ $\left({ }_{-}{ }_{-}\right), r=0.01(--), r=0$ (_.._). (b) $w$ varying, $w=0.1(-), w=0.05(--), w=0.03\left(\right.$ _ $_{-}$),$w=0.01(--)$, $w=0$ (_.._). (c) q varying, $q=0.5(-), q=0.3(---), q=0.1\left({ }_{-} \cdot{ }_{-}\right), q=0.05(--), q=0\left({ }_{-} \cdot{ }_{-}\right)$. (d) $p$ varying, $p=0.2(-), p=0.15(--), p=0.1\left({ }_{-} \cdot{ }_{-}\right), p=0.05(--), p=0\left({ }_{-} \cdot{ }_{-}\right)$.

When we consider a policy that ignores defects, true or false (lines 17-20), we again see a polarization of $\mathrm{ABR}$ and $\mathrm{PI}$, but that when inspection is preferred (smaller $r$ ) it is more frequent (smaller T), and the cost-rate is larger (compare 8 with 19). These observations affirm those of Cavalcante et al. (2011) regarding the efficacy of a policy that allows detection of a defective state. Our final point relates to perfect inspection (line 21) and perfect maintenance (line 22). In both cases, the $M T$ policy is cost-optimal. However, a more interesting overall observation is how dramatic is the effect of imperfection (low quality) - the cost-rate for the base case is more than double. This demonstrates that quality of maintenance has a very significant impact on cost (and losses).

Finally we note that when the optimal $M T$ policy warrants inspections, $M T$ is large, allowing fuller use of component lives. 


\subsubsection{The utility of the general model}

The aspects of maintenance quality considered in our model have already been addressed separately in other studies. The uniqueness of our study lies in considering these aspects together. Then the extent to which simpler models provide poorer guidance for maintainers can be studied.

In Table 2 we present the marginal increased cost of implementing a sub-optimal policy. The base line cost-rate is the cost-rate of the optimal policy $\left(M^{*}, T^{*}\right)$ under the full model. The full model accounts for all aspects of maintenance quality. We then assume a reduced model (that omits an aspect of maintenance quality) and determine the optimal policy $\left(M^{\prime}, T^{\prime}\right)$ under this reduced model. We then calculate the cost-rate considering all aspects of maintenance quality (full model) at $\left(M^{\prime}, T^{\prime}\right)$. The marginal increased cost $\left(100 \times\left\{C_{\infty}\left(M^{\prime}, T^{\prime}\right)-C_{\infty}\left(M^{*}, T^{*}\right)\right\} / C_{\infty}\left(M^{*}, T^{*}\right) \%\right)$ is then termed the "cost of ignorance".

Table 2. Marginal increased cost of sub-optimal policies

\begin{tabular}{|c|c|c|c|c|c|c|c|c|c|c|c|c|}
\hline \multicolumn{13}{|c|}{ Model assumed } \\
\hline & & \multicolumn{4}{|c|}{ Quality parameters } & \multicolumn{4}{|c|}{$\begin{array}{l}\text { Cost-optimal policy under } \\
\text { the reduced model }\end{array}$} & \multicolumn{2}{|c|}{$\begin{array}{l}\text { Cost-rate and } \\
\text { loss-rate of } \\
\text { policy under } \\
\text { full model }\end{array}$} & $\begin{array}{c}\text { Cost of } \\
\text { ignorance } \\
\%\end{array}$ \\
\hline & & $p$ & $w$ & $q$ & $r$ & $M$ & $T$ & $C_{\infty}$ & $S h_{\infty}$ & $C_{\infty}$ & $S h_{\infty}$ & \\
\hline \multirow{6}{*}{$\begin{array}{ll}3 & D_{0} \\
0 & 0 \\
0 & 0 \\
\frac{0}{D} \\
\frac{D}{n} & 0 \\
0\end{array}$} & Full model & 0.10 & 0.05 & 0.3 & 0.05 & 1 & 0.785 & 1.856 & 1.659 & 1.856 & 1.659 & 0 \\
\hline & No heterogeneity & 0 & 0.05 & 0.3 & 0.05 & 1 & 1.239 & 1.072 & 1.419 & 2.404 & 1.464 & $29.5 \%$ \\
\hline & No defect induction & 0.1 & 0.05 & 0.3 & 0 & 17 & 0.178 & 1.051 & 1.288 & 2.034 & 1.384 & $9.6 \%$ \\
\hline & No classification errors & 0.1 & 0 & 0 & 0.05 & 25 & 0.146 & 1.656 & 1.295 & 2.032 & 1.413 & $9.5 \%$ \\
\hline & Perfect inspection & 0.1 & 0 & 0 & 0 & 20 & 0.164 & 0.818 & 1.197 & 2.026 & 1.393 & $9.2 \%$ \\
\hline & Perfect maintenance & 0 & 0 & 0 & 0 & 15 & 0.192 & 0.749 & 1.201 & 2.049 & 1.376 & $10.4 \%$ \\
\hline
\end{tabular}

Ignoring heterogeneity is the most costly (29.5\%). Ignoring classification errors and induced defects is less costly (about 10\%). Interestingly, complete ignorance (assuming perfect maintenance) is much less costly than ignoring heterogeneity alone. This is because for the former the policy recommends inspection, and inspections are useful to deal with early defects due weak items. In addition, complete ignorance of inspection quality and ignorance of defect induction or classification errors cost approximately the same. This is because, in each case, the recommended policy is frequent inspection, thus mitigating for component heterogeneity.

\subsubsection{Investing in higher quality maintenance}

Maintenance quality can be improved in various ways, e.g. through staff training and development and use of new supportive technology. In general, quality improvement requires investment, and in Table 3 we highlight where investments may be beneficial. Thus, comparing the base case (line 1) with cases in which the defect classification accuracy is improved (lines 2 and 3), we see that a $25 \%$ increase in the cost of inspection $\left(c_{\mathrm{I}}\right)$ to reduce $w$ by $80 \%$ is worthwhile (since the cost-rate is smaller for line 2 than line 1), but a 50\% increase is not. Regarding defect induction, investment is more beneficial (lines 1 and lines 4-6), where broadly a $100 \%$ increase in $c_{\mathrm{I}}$ is worthwhile to achieve a $50 \%$ reduction in $r$.

Other effects can be observed. To reduce $p$ (heterogeneity) requires investment in replacements (through $c_{\mathrm{G}}$ etc). A $10 \%$ increase in replacement cost is worthwhile to achieve a $50 \%$ reduction in heterogeneity but not $20 \%$ (lines 11,12 ). This analysis is brief, but the scope for determining where to invest or for supporting a case for investment is demonstrated. Broadly, investment in reducing defect 
induction is the most cost-effective investment. The loss-rate effects are broadly to the contrary and so this indeed provides a useful additional criterion to guide decision-making.

Table 3. An analysis of the benefit of investment in higher quality maintenance.

\begin{tabular}{lcccccccccccccc}
\hline & \multicolumn{1}{c}{ Quality parameters } & \multicolumn{4}{c}{ Costs } & \multicolumn{4}{c}{ Optimum policy } \\
\hline Case & $p$ & $w$ & $q$ & $r$ & \multicolumn{1}{c}{$c_{\mathrm{I}}$} & \multicolumn{1}{c}{$c_{\mathrm{G}}$} & $c_{\mathrm{D}}$ & $c_{\mathrm{F}}$ & $M$ & \multicolumn{1}{c}{$T$} & $M T$ & $C_{\infty}$ & $S h_{\infty}$ \\
\hline 1 Base case & 0.10 & 0.05 & 0.30 & 0.05 & 0.04 & 1 & 1.40 & 2 & 1 & 0.785 & 0.785 & 1.856 & 1.659 \\
2 Improve classification & 0.10 & 0.01 & 0.10 & 0.05 & 0.05 & 1 & 1.40 & 2 & 24 & 0.151 & 3.624 & 1.816 & 1.314 \\
3 Improve classification & 0.10 & 0.01 & 0.10 & 0.05 & 0.06 & 1 & 1.40 & 2 & 21 & 0.161 & 3.381 & 1.879 & 1.312 \\
4 Reduce defect induction & 0.10 & 0.05 & 0.30 & 0.01 & 0.06 & 1 & 1.40 & 2 & 15 & 0.192 & 2.880 & 1.359 & 1.305 \\
5 Reduce defect induction & 0.10 & 0.05 & 0.30 & 0.01 & 0.08 & 1 & 1.40 & 2 & 13 & 0.211 & 2.743 & 1.457 & 1.303 \\
6 Reduce defect induction & 0.10 & 0.05 & 0.30 & 0.02 & 0.08 & 1 & 1.40 & 2 & 13 & 0.207 & 2.691 & 1.662 & 1.324 \\
7 Reduce defect induction & 0.10 & 0.05 & 0.30 & 0.03 & 0.08 & 1 & 1.40 & 2 & 13 & 0.203 & 2.639 & 1.864 & 1.344 \\
8 Improve inspection & 0.10 & 0.01 & 0.10 & 0.01 & 0.12 & 1 & 1.40 & 2 & 11 & 0.244 & 2.684 & 1.448 & 1.251 \\
9 Improve inspection & 0.10 & 0.01 & 0.10 & 0.02 & 0.12 & 1 & 1.40 & 2 & 12 & 0.229 & 2.748 & 1.645 & 1.263 \\
10 Improve inspection & 0.10 & 0.01 & 0.10 & 0.03 & 0.12 & 1 & 1.40 & 2 & 12 & 0.224 & 2.688 & 1.837 & 1.281 \\
11 Reduce weak items & 0.05 & 0.05 & 0.30 & 0.05 & 0.04 & 1.10 & 1.54 & 2.20 & 1 & 0.954 & 0.954 & 1.670 & 1.546 \\
12 Reduce weak items & 0.05 & 0.05 & 0.30 & 0.05 & 0.04 & 1.50 & 2.10 & 3 & 1 & 1.042 & 1.042 & 2.082 & 1.506 \\
13 Joint improvement & 0.05 & 0.01 & 0.10 & 0.01 & 0.12 & 1.50 & 2.10 & 3 & 11 & 0.255 & 2.805 & 1.648 & 1.237 \\
14 Joint improvement & 0.05 & 0.01 & 0.10 & 0.02 & 0.12 & 1.50 & 2.10 & 3 & 11 & 0.251 & 2.761 & 1.867 & 1.253 \\
15 Joint improvement & 0.05 & 0.01 & 0.10 & 0.03 & 0.12 & 1.50 & 2.10 & 3 & 12 & 0.238 & 2.856 & 2.084 & 1.263 \\
16 Joint improvement & 0.05 & 0.01 & 0.10 & 0.01 & 0.12 & 2 & 2.80 & 4 & 12 & 0.251 & 3.012 & 1.903 & 1.228 \\
\hline
\end{tabular}

\section{CONCLUSION}

In this paper, we study a mixed policy of inspection and preventive replacement for a singlecomponent protection system. Failures of the system are modelled with the delay time concept. Component lifetimes (specifically times to defect arrival) arise from a mixture distribution (heterogeneity). The system is inspected to determine its state, and an inspection may classify a good system as defective (false positive) and a defective system as good (false negative) (misclassification errors). An inspection may itself change the state of the system from good to defective (defect induction). These aspects of maintenance quality (heterogeneity, misclassification, defect induction) are parameterized, and their effects upon the cost-rate and the production loss-rate of a near-periodic inspection and replacement policy are studied. Isolation valves in a water distribution system motivate the analysis. There are important implications for practice because the model provides decision support for prioritizing quality improvement.

During the data collection for the case study, engineers recognized defect induction and misclassification as significant concerns, but their extent was unknown. We quantify these, and find that reducing defect induction is the priority for investment, although this in conjunction with the reduction of misclassification has even greater benefit. This demonstrates the value of the model we develop.

Careful estimation of quality parameters is required to make full use of our model. Methods for elicitation of maintenance modelling parameters exist, e.g. snapshot modelling (Christer and Whitelaw, 1983), interval method (Beraldi et al., 2013), but there are difficulties not least because the elicitation of quality parameters is equivalent to asking maintainers to how well they do their job. In relation to this, the quality parameters in our model may be suitable performance indicators for a third-party maintenance contract (Brito et al., 2010). Finally, for multiple protection systems with varying criticalities in a network, investment prioritization would need to consider not only broad 
quality improvement but also targeted interventions for the most critical systems (Scarf and Martin, 2001).

The model has been developed for protection systems like valves, whose operation is binary (e.g. open or closed). It may be interesting, in future work, to develop corresponding models for protection systems that may provide partial protection, as a result of deterioration of the protection system, in the event of a demand.

Acknowledgements: The work of Cristiano Cavalcante has been supported by CNPq (Brazilian Research Council). The work of Alexandre Alberti has been supported by FACEPE (Foundation for Science and Technology of the State of Pernambuco). The work of Phil Scarf has been supported by the College of Science, King Saud University, Saudi Arabia.

\section{REFERENCES}

Ascher H, Feingold H (1984) Repairable Systems Reliability. Marcel Dekker, New York.

Aven T, Jensen, U (1999) Stochastic Models in Reliability. Springer-Verlag, New York.

Baraldi P, Compare M, Zio E (2013) Maintenance policy performance assessment in presence of imprecision based on Dempster-Shafer Theory of Evidence. Information Sciences 245, 112-131.

Berrade MD, Cavalcante CAV, Scarf PA (2012) Maintenance scheduling of a protection system subject to imperfect inspection and replacement. European Journal of Operational Research 218, 716-725.

Berrade MD, Scarf PA, Cavalcante CAV (2015) Some insights into the effect of maintenance quality for a protection system. IEEE Transactions on Reliability 64, 661-672.

Berrade MD, Scarf PA, Cavalcante CAV, Dwight RA (2013) Imperfect inspection and replacement of a system with a defective state: A cost and reliability analysis. Reliability Engineering and System Safety 120, 80-87.

Brito AJM, de Almeida Filho AT, de Almeida AT (2010) Multi-criteria decision model for selecting repair contracts by applying utility theory and variable interdependent parameters. IMA Journal of Management Mathematics 21, 349-361.

Cavalcante CAV, Scarf PA, Almeida AT (2011) A study of a two-phase inspection policy for a preparedness system with a defective state and heterogeneous lifetime. Reliability Engineering and System Safety 96: 627-635.

Cavalcante CAV, Scarf PA, Berrade MD (2017) A study of imperfect inspection of a protection system. Working paper.

Christer AH (1999) Developments in delay time analysis for modelling plant maintenance. Journal of the Operational Research Society 50, 1120-1137.

Christer AH, Scarf PA (1994) A robust replacement model with applications to medical equipment. Journal of the Operational Research Society 45, 261-275.

Christer AH, Whitelaw J (1983) An Operational Research approach to breakdown maintenance: problem recognition. The Journal of the Operational Research Society 34, 1041-1052.

Dagg R, Newby M (1998) Optimal overhaul intervals with imperfect inspection and repair. IMA Journal of Management Mathematics 9, 381-391.

De Jonge B, Dijkstra AS, Romeijnders W (2015) Cost benefits of postponing time-based maintenance under lifetime distribution uncertainty. Reliability Engineering and System Safety 140, 15-21.

Driessen JPC, Peng H, Van Houtum GJ (2017) Maintenance optimization under non-constant probabilities of imperfect inspections. Reliability Engineering and System Safety 165, 115-123.

Dwight RA, Scarf PA, Gordon P (2012) Dynamic maintenance requirements analysis for asset management. In Advances in Safety, Reliability, and Risk Management (Berenguer, Gralland, and Guedes Soares, eds), Taylor and Francis, London, pp. 847-852. 
Flage R (2014) A delay time model with imperfect and failure-inducing inspections. Reliability Engineering \& System Safety 124, 1-12.

Giustolisi O, Savic D (2010) Identification of segments and optimal isolation valve system design in water distribution networks. Urban Water Journal 7, 1-15.

Goulter C, Coals AV (1986) Quantitative approaches to reliability assessment in pipe networks. Journal of Transportation Engineering 122, 287-301.

Jia X, Christer AH (2002) A periodic testing model for a preparedness system with a defective state. IMA Journal of Management Mathematics 13, 39-49.

Jun H, Loganathan GV, Deb AK, Grayman W, Snyder J (2007) Valve distribution and impact analysis in water distribution systems. Journal of Environmental Engineering 133, 790-799.

Kim JH, Mays LW (1994) Optimal rehabilitation model for water-distribution systems. Journal of Water Resources Planning and Management 120, 674-692.

Liu H, Walski T, Fu G, Zhang C (2017) Failure impact analysis of isolation valves in a water distribution network. Journal of Water Resources Planning and Management 143(7).

Marlow DR, Beale DJ, Mashfort JS (2012) Risk-based prioritization and its application to inspection of valves in the water sector. Reliability Engineering and System Safety 100, 67-74.

Okumura S, Jardine AKS, Yamashina H (1996) An inspection policy for a deteriorating single-unit system characterized by a delay-time model. International Journal of Production Research 34, 2441-2460.

Scarf PA (1997) On the application of mathematical models in maintenance. European Journal of Operational Research 99, 493-506.

Scarf PA, Cavalcante CAV (2012) Modelling quality in replacement and inspection maintenance. International Journal of Production Economics 135, 372-381.

Scarf PA, Cavalcante CAV, Dwight RA, Gordon P (2009) An age-based inspection and replacement policy. IEEE Transactions on Reliability 58, 641-648.

Scarf PA, Martin H (2001) A framework for maintenance and replacement of a network structured system. International Journal of Production Economics 69, 287-296.

Selvik JT, Aven T (2011) A framework for reliability and risk centered maintenance. Reliability Engineering \& System Safety 96, 324-331.

Vaurio JK (1995) Unavailability analysis of periodically tested standby components. IEEE Transactions on Reliability 44, 512-517.

Vaurio JK (199() Availability and cost functions for periodically inspected preventively maintained units. Reliability Engineering and System Safety 63, 133-140.

Walski TM (1993) Water distribution valve topology for reliability analysis. Reliability Engineering and System Safety 42, 21-27.

Zyl JV (2014) Introduction to Operation and Maintenance of Water Distribution Systems. Republic of South Africa, Water Research Commission. 